\title{
Erratum to: Maternal inheritance of mitochondrial genomes and complex inheritance of chloroplast genomes in Actinidia Lind.: evidences from interspecific crosses
}

\author{
Dawei $\mathrm{Li}^{1} \cdot \mathrm{Xiaoqiong}_{\mathrm{Qi}^{2}} \cdot{\mathrm{Xinwei} \mathrm{Li}^{1} \cdot \mathrm{Li} \mathrm{Li}^{1} \cdot \text { Caihong Zhong }}^{1} \cdot$ Hongwen Huang ${ }^{1,3}$
}

Published online: 9 April 2015

(C) Springer-Verlag Berlin Heidelberg 2015

\section{Erratum to: Mol Genet Genomics (2013) 288(3-4):101-110 DOI 10.1007/s00438-012-0732-6}

In the original publication, one of the grant project numbers, NSF of China grant (Y211121N01) in the Acknowledgments section has been incorrectly published.

The corrected Acknowledgments should read as:

We would like to thank Datson Paul (The New Zealand Institute for Plant and Food Research Ltd) for his critical comments and suggestions, Quan Zhang (Peking University, China) and Xiaohong Yao for their helpful discussions, and Fei Han and Xiaoli Liu for help with crossing experiments and cultivation. This project was funded by the project (Y152091t02) of 'Key Laboratory of Plant Germplasm Enhancement and Specialty Agriculture, Wuhan Botanical Garden, Chinese Academy of Sciences' and NSF of China grant (31101520).

The authors apologize for this error.

The online version of the original article can be found under doi:10.1007/s00438-012-0732-6.

Caihong Zhong

zhongch1969@163.com

$\triangle$ Hongwen Huang

1 Key Laboratory of Plant Germplasm Enhancement and Specialty Agriculture, Wuhan Botanical Garden, Chinese Academy of Sciences, Wuhan 430074, Hubei, People's Republic of China

2 School of Chemistry and Life Science, Hubei University of Education, Wuhan Hi-Tech Zone, Wuhan 430205, Hubei, People's Republic of China

3 Key Laboratory of Plant Resources Conservation and Sustainable Utilization, South China Botanical Garden, Chinese Academy of Sciences, Guangzhou 510650,

Guangdong, People's Republic of China 